\title{
Surface tension and viscosity of blood
}

\author{
Wesolowski Adam ${ }^{1} \&$ Mlynarczak Anna ${ }^{1}$ \\ July 4, 2019 \\ 1.Department of Biochemistry Wroclaw Medical University
}

\begin{abstract}
1 Abstract
Blood has certain significant physical properties, which the authors investigated. This paper provides values of surface tension and viscosity of blood at $37^{\circ} \mathrm{C}$, as well as relationship between them plotted on a cross-plot. By applying HagenPoiseuille equation and Jurin's law to the experimentally obtained data, the authors calculated with great accuracy viscosity and surface tension of blood. Evaluating both of the properties using described methods required from the authors to know the density of investigated liquid, which was found to be on average $\rho=1063,56 \frac{\mathrm{kg}}{\mathrm{m}^{3}}$. The authors took blood samples from 30 healthy subjects and determined aforementioned physical properties. There has been made a distinction in regard of a sex of the blood donor. Results indicate that both surface tension and viscosity are independent from sex of a subject, as well as indicate that there is no correlation between viscosity and surface tension of blood at $37^{\circ} \mathrm{C}$. Average values of surface tension and viscosity were found to be $\sigma=5,241 \pm 0,262 \cdot 10^{-2} \frac{\mathrm{J}}{\mathrm{m}^{2}}$ and $\eta=3,352 \pm$ $0,360 \cdot 10^{-3} \mathrm{~Pa} \cdot \mathrm{s}$, respectively.
\end{abstract}

Key words: surface tension, viscosity, blood. 


\section{Introduction}

Importance of studying blood properties is indisputable. A deep red fluid that flows out taking life with itself aroused fascination from the very beginning of human's cognition. In the light of nowdays advanced medicine it is still among the most commonly discussed connective tissues and it spreads a considerable interest due to it's importance. Since humans consist in major part of blood, it requires thoroughgoing analysis and investigations. With this in mind, the authors decided to make a contribution to broadening the knowledge about the one of the most important human's connective tissues. Despite the whole blood being already deeply investigated, neither surface tension nor viscosity have fixed values, and those properties still require profound investigations. There do exist many works related to the aforementioned properties $^{1-10}$, nevertheless to the best of the author's knowledge not much work has been done to investigate whether there exist a correlation between surface tension and viscosity for colloids such as blood and organic fluids at all, beyond those by Schonhorn ${ }^{2}$ and Pelofsky ${ }^{10}$, which give reasonable results for most of fluids. However, there have been made no attempt to investigate this relationship for the human blood. In general, all works are empirical and there is still no widely accepted theoretical model connecting viscosity and surface tension. Thereby, the authors depicted the relationship resultant from their investigation for the human blood on a cross-plot for the whole blood at $37^{\circ} \mathrm{C}$. The authors reckon the importance of surface tension and viscosity of blood is underestimated as they do not appear in many medical books and coursebooks, despite being a source of valuable information about an organism ${ }^{1,4,7,8,9}$. Thus the authors felt determined and motivated to conduct all experiments and basing on the obtained data evaluate the values of aforementioned physical properties and investigate the probable correlation between them.

\section{Materials and methods}

Whole blood was taken from 30 volunteers, among whom there were 15 men and 15 women. Blood was collected into heparinized test tubes and investigated within 5 hours after collection. Viscosity, surface tension and density were experimentally evaluated. Each property is dependent from many variables including temperature, protein content and haematocrit. There have not been made distinctions in regard to haematocrit and protein content, though temperature was fixed at $37^{\circ} \mathrm{C}$ and both surface tension and viscosity have been evaluated under this condition. Glass capillary tubes of diameter $1 \mathrm{~mm}$ and $2,4 \mathrm{~mm}$ were used during investigations of surface tension and viscosity, respectively. Blood was weighted on a scale of 0,01 gram measuring accuracy.

Viscosity of blood has been calculated using HagenPoiseuille equation which can be stated as: $\eta=\frac{t \pi r^{4} \Delta p}{8 V l}$, where:

$\eta$ - is the dynamic viscosity of the liquid,

$\mathrm{t}$ - is the time that it takes the volume $\mathrm{V}$ to flow through the capillary tube of length 1 and radius $r$ under average pressure $\Delta p$. Needed variables were measured by Poiseuille method ${ }^{11}$ of letting liquid to freely flow through a capillary tube. In this experiment the tube was $5 \mathrm{~cm}$ long and the average pressure between the ends of the tube was estimated to be $260 P a$. Method was based on attaching the capillary tube at the opening of the properly caulked test tube, then turning the system upside down in order to enable required laminar flow through the capillary. This procedure was done swiftly, with previously the test tube being, gently turned upside down a few times, to minimalize the effect of aggregation of red cells on whole blood viscosity ${ }^{7}$. Hagen- Poiseuille equation ${ }^{12,13}$ assumes laminar flow, the authors checked if this condition was satisfied. The boundary value of Reynolds number ${ }^{14}[\operatorname{Re}=$ $\left.\frac{\rho v l}{\eta}\right]$ for the whole blood is thought to be around $2000^{14,15}$, below this critical value the flow is supposed to be laminar. Though in our case Re has slightly exeeded this value it was shown that the laminar flow can be maintained up to the value of $\operatorname{Re}=12000 .{ }^{16}$ In fact we can assume with high probability that the flow was indeed laminar in our case. In determining the surface tension of blood using Jurin's law ${ }^{17,18}$, which is followed by the equation: $\sigma=\frac{\rho g h r}{2 \cos \theta}$, where:

$\sigma$ - is the surface tension of liquid,

$\rho$ - is the mass density,

$\mathrm{h}$ - height at which fluid rose in capillary of radius $\mathrm{r}$,

$\cos \theta$ - is the contact angle between the fluid and the wall of the capillary tube,

$\mathrm{g}$ - is gravitational acceleration equal to $9,81 \frac{\mathrm{m}}{\mathrm{s}^{2}}$, it was necessary to measure the height at which the liquid rose in the capillary tube. The angle was evaluated to be $20^{\circ} \pm 10^{\circ}$. Process of the measurement looked the following - the sample of blood was poured into the Petri dish, the single capillary tube was perpendicularly steeped into the liquid and held still for 30 seconds. After that the tube was gently placed next to the ruler for the height of the liquid to be measured. As no measurement was aimed directly into the wanted values, the authors were obligated to use the exact differential method while coping with measurement error. This research was approved by Bioethical Committee at Lower Silesian Medical Association on 10th of April 2019. Number of the approval is 2/PN/2019.

\section{Results}

Average value of surface tension and viscosity are 5, 278 . $10^{-2} \frac{\mathrm{J}}{\mathrm{m}^{2}}, 5,204 \cdot 10^{-2} \frac{\mathrm{J}}{\mathrm{m}^{2}}$ and $3,293 \cdot 10^{-3} \mathrm{~Pa} \cdot \mathrm{s}, 3,411$. $10^{-3} \mathrm{~Pa} \cdot \mathrm{s}$ among female and male samples, respectively. Exact results are presented in tables (see Tab.1 and Tab.2). It can be claimed that those values are independent from the sex of a subject. Average density of blood was found to be $1063,56 \frac{\mathrm{kg}}{\mathrm{m}^{3}}$. No unexpected values of neither surface tension and viscosity nor density occurred. Relationship between these properties is shown on a cross-plot (see. Fig.1).Viscosity assume many different values at the same level of surface tension, thus the authors conclude there is no correlation viscosity-surfce tension for human whole blood at $37^{\circ} \mathrm{C}$. There are some points corresponding to higher and lower surface tension, this might be mainly the result of the measurement accuracy, and should not be the subject of concerns. 


\section{Discussion}

Surface tension can be defined as the work that should be done to the surface of the liquid to rip this surface, thereby the dimension of surface tension is $\frac{J}{\mathrm{~m}^{2}}$ in SI units. There are numerous definitions of surface tension, some describe it as a tendency to shrink so that the ratio $\frac{\text { syrface }_{\text {area }}}{\text { volume }}$ is as small as possible, the others add the description that the shrinking is caused by cohesive forces.

The authors were able to find only two works that provide values of surface tension of blood. One of them is by Hrncír and Rosina ${ }^{8}$, which additionally shows the importance of surface tension in functioning of human organism. According to the second research done by Rosina et $\mathrm{al}^{9}$ that is based on a ring method, there is a linear dependence between temperature and surface tension of blood, and is given by equation $\sigma=(-0.473 t+70.105)$. $10^{-3} \frac{\mathrm{N}}{\mathrm{m}}$, where $\mathrm{t}$ stands for temperature. Note that the dimension $\frac{N}{m}$ is identical to $\frac{J}{m^{2}}$. If this equation holds the value of surface tension of blood at $37^{\circ} \mathrm{C}$ should be around $5,26 \cdot 10^{-2}$ what definitely agrees with our results.

Viscosity is dependent from a wide set of variables, while surface tension mostly depends on temperature and chemical compounds constituting to a liquid. According to Pries et $\mathrm{al}^{3}$ viscosity depends also on haematocrit and the diameter of the pipe, the liquid is flowing through. The latter is often referred as Fahraeus-Lindqvist effect ${ }^{19,20}$, which was discovered by Fåhraeus and Lindqvist ${ }^{21}$ themselves in 1930. According to their work viscosity of blood highly decrease with the reduction of the radius of the capillary tube, the effect becomes significant below the diameter of about $0.3 \mathrm{~mm}$. Thereby the the pipe used in the experiments does not significantly influence the results. Replogle et $\mathrm{al}^{7}$ point out that fibrinogen has a considerable impact on the blood viscosity as it cause red cells to aggregate. According to his research at high shear rates, blood behaves as a Newtonian fluid, whereas normally is treated as non-Newtonian. Tao and Huang ${ }^{5}$ prove in their work that viscosity of blood can be changed by applying the magnetic field and thus can be regulated in cases of dangerously abnormal deviations. It can be seen how important role in human organism one viscosity plays, and how strongly it affects health state. Thanks to the result of our work, values of eponymous physical properties can be compared to others, and the reliability of the used methods assessed. On the cross-plot (see Fig.1) there are many different values of viscosity for the same value of surface tension, that is probably due to the factors that influence viscosity but not surface tension. Moreover, what stems from our research, the Pelofsky's equation $^{10}\left(\sigma=A e^{\frac{B}{\eta}}\right)$ does not hold for human blood at $37^{\circ} \mathrm{C}$. The authors supported by the results of their work feel confident enough to put forward a claim that viscosity is completely independent from surface tension for the whole blood at $37^{\circ} \mathrm{C}$. Supposing this claim is true, it can have remarkable implications in field of pharmacy. Moreover, the authors suggest to take under consideration whether there exist a correlation between surface tension and viscosity in a wide range of temperatures, as the results might differ.

\section{Acknowledgments}

At the very outset, the authors offer their sincere gratitude to the Dr. Kamillia Stach for support at every point of work, valuable suggestions and inspiring guidance during pre- experimental time of preparations.

The authors feel themselves fortunate enough in completing this work by courtesy of head of the Department of Biochemistry prof. hab. dr. Andrzej Gamian who in his kindness provided the laboratory facilities for conducting experiments.

The authors wishes to express profused thanks to Dr. Tomasz Wesołowski for organisational support. The authors are indebted to their beloved parents, as without their contribution this work could not have been done by the authors. Adam Wesolowski would like to express deep sense of gratitude to his grandfather Prof. Piotr Wesołowski, who had a profund contribution to his interest in science.

\section{References}

[1] Raymond Ma, Smith Er, Liesega J . The physical properties of blood - forensic considerations.Sci Justice 1996; 36: 153-1 60

[2]Schonhorn Harold. Surface Tension- Viscosity Relationship for Liquids. Journal of Chemical \& Engineering Data,1967; volume 12, number 4, pages, 524-525.

[3] Pries A. R., Neuhaus D., Gaehtgens P.. Blood viscosity in tube flow: dependence on diameter and hematocrit. AJP - Heart and Circulatory Physiology. 1992; Volume 263: H1770 - Dec 1,9 pages

[4] Dormandy J A. "Clinical significance of blood viscosity." Annals of the Royal College of Surgeons of England, 1967; vol. 47,4: 211-28.

[5] Tao Rongjia, Huang Ke. Reducing blood viscosity with magnetic fields. Physical review. E, Statistical, nonlinear, and soft matter physics. 2011; 84. 011905. 10.1103/PhysRevE.84.011905..

[6] Rosenson R.S., Mccormick A., Uretz E.F.. Distribution of blood viscosity values and biochemical correlates in healthy adults. Clinical chemistry, 1994; 428 Pt 1, 1189-95 [7] Robert L. Replogle M.D., Meiselman J. Herbert Sc.D., Merrill W. Edward Sc.D. Clinical Implications of Blood Rheology Studies. Circulation,1967; vol.36 july

[8] Hrncír E., Rosina J. Surface tension of blood. Physiological research. 1997;46(4):319-21.

[9] Rosina J. Kvasnak Eugen, Suta Daniel, Kolárová H., Malek Jiri, Krajci L. Temperature dependence of blood surface tension. Physiological research. 2007;56 Suppl 1. S93-8.

[10] Pelofsky A. H. Surface Tension-Viscosity Relation for Liquids. Journal of Chemical Engineering Data 1966; 11 (3), 394-397

[11] Marek Vilipuu Tallinn University of Technology, Division of Physics www.ttu.ee/public/m/Marek Vilipuu/FI_doks/FI/FI_labs_in_english/ Experiment_12.pdf

[12] Catherine Loudon, Katherine McCulloh. Application of the Hagen-Poiseuille Equation to Fluid Feeding through Short Tubes, Annals of the Entomological Society of America, 1999; Volume 92, Issue 1, 1 January, Pages 
153-158

[13] Pfitzner, J. P.. "Poiseuille and his law." Anaesthesia. $1976 ; 312: 273-5$.

[14]Tom Benson NASA Glenn Research Center WWW. grc. nasa. gov/WWW/BGH/reynolds. html 2014;

[15] Carl Rod Nave Department of Physics and Astronomy Georgia State University hyperphysics.phy-astr.gsu. edu/hbase/pturb. html

[16] Trinh Khanh Tuoc. On The Critical Reynolds Number For Transition From Laminar To Turbulent Flow.2010 [17] Rodríguez-Valverde Miguel A., Tirado-Miranda Maria. Derivation of Jurin's law revisited. European Journal of Physics. 2010; 32. 49. 10.1088/0143-0807/32/1/005.

[18] Alexandre Ponomarenko, David Quéré, Christophe Clanet. A universal law for capillary rise in corners.Journal of Fluid Mechanics, Cambridge University Press (CUP), 2011; 666 (january), pp.146154.10.1017/s0022112010005276. hal-00994488.

[19] K.Snyder Gregory. Erythrocyte evolution: The significance of the Fåhraeus-Lindqvist phenomenon.Respiration Physiology. 1973; Volume 19, Issue 3, December, Pages 271-278

[20] James H. Barbee, Giles R.Cokelet. The Fahraeus effect. Microvascular research.1971; 3. 6-16. 10.1016/00262862(71)90002-1.

[21] Fåhraeus Robin, Lindqvist Torsten. The viscosity of the blood in narrow capillary tubes. Physiology.01 March 1931 received for publication 06 December 1930

\begin{tabular}{ccc}
\hline Sex and volunteer's number & Time $[s]$ & $\eta=[m P a \cdot s]$ \\
\hline$F_{1}$ & 7,60 & 3,797 \\
$F_{2}$ & 7,72 & 3,857 \\
$F_{3}$ & 7,06 & 3,527 \\
$F_{4}$ & 7,24 & 3,617 \\
$F_{5}$ & 7,07 & 3,532 \\
$F_{6}$ & 6,84 & 3,418 \\
$F_{7}$ & 6,77 & 3,383 \\
$F_{8}$ & 5,58 & 2,788 \\
$F_{9}$ & 6,53 & 3,263 \\
$F_{10}$ & 7,80 & 3,897 \\
$F_{11}$ & 6,72 & 3,358 \\
$F_{12}$ & 5,18 & 2,588 \\
$F_{13}$ & 5,55 & 2,773 \\
$F_{14}$ & 5,10 & 2,548 \\
$F_{15}$ & 6,11 & 3,053 \\
& & \\
$M_{1}$ & 6,80 & 3,398 \\
$M_{2}$ & 6,98 & 3,487 \\
$M_{3}$ & 6,93 & 3,462 \\
$M_{4}$ & 6,60 & 3,298 \\
$M_{5}$ & 6,87 & 3,433 \\
$M_{6}$ & 7,75 & 3,872 \\
$M_{7}$ & 7,35 & 3,672 \\
$M_{8}$ & 6,24 & 3,118 \\
$M_{9}$ & 7,68 & 3,837 \\
$M_{10}$ & 7,43 & 3,712 \\
$M_{11}$ & 6,31 & 3,652 \\
$M_{12}$ & 5,88 & 3,403 \\
$M_{13}$ & 5,52 & 3,2638 \\
$M_{14}$ & 6,24 & 3,118 \\
$M_{15}$ & & \\
& & \\
\hline & 635 \\
\hline
\end{tabular}

Table 1: Table with the values of time of blood flow through the capillary tube, and viscosity corresponding to that time. $M_{i}$ and $F_{i}$ stands for Male and Female, respectively, ' $i$ ' denotes the number of the subject. 


\begin{tabular}{cccr}
\hline Sex and volunteer's number & Height $[\mathrm{m}]$ & $\sigma=\left[\frac{P a}{m} \cdot 10^{-2}\right]$ \\
\hline$F_{1}$ & 0,018 & 5,000 & Th \\
$F_{2}$ & 0,018 & 5,000 & \\
$F_{3}$ & 0,019 & 5,278 & \\
$F_{4}$ & 0,018 & 5,000 & $\mathbf{9}$ \\
$F_{5}$ & 0,019 & 5,278 & \\
$F_{6}$ & 0,020 & 5,556 & A \\
$F_{7}$ & 0,020 & 5,556 & the \\
$F_{8}$ & 0,019 & 5,278 & data \\
$F_{9}$ & 0,019 & 5,278 & \\
$F_{10}$ & 0,020 & 5,556 \\
$F_{11}$ & 0,019 & 5,278 \\
$F_{12}$ & 0,020 & 5,556 \\
$F_{13}$ & 0,019 & 5,278 \\
$F_{14}$ & 0,019 & 5,278 \\
$F_{15}$ & 0,019 & 5,278 \\
$M_{1}$ & & \\
$M_{2}$ & 0,019 & 5,278 \\
$M_{3}$ & 0,018 & 5,000 \\
$M_{4}$ & 0,020 & 5,556 \\
$M_{5}$ & 0,019 & 5,278 \\
$M_{6}$ & 0,019 & 5,278 \\
$M_{7}$ & 0,020 & 5,556 \\
$M_{8}$ & 0,019 & 5,278 \\
$M_{9}$ & 0,019 & 5,278 \\
$M_{10}$ & 0,019 & 5,278 \\
$M_{11}$ & 0,018 & 5,000 \\
$M_{12}$ & 0,017 & 4,722 \\
$M_{13}$ & 0,019 & 5,278 \\
$M_{14}$ & 0,018 & 5,000 \\
$M_{15}$ & 0,019 & 5,278 \\
& 0,018 & 5,000 \\
\hline
\end{tabular}

Table 2: Table with values of height reached by blood in the capillary tube and corresponding values of surface tension. $M_{i}$ and $F_{i}$ stand for Male and Female, respectively, ' $i$ ' denotes the number of the subject.

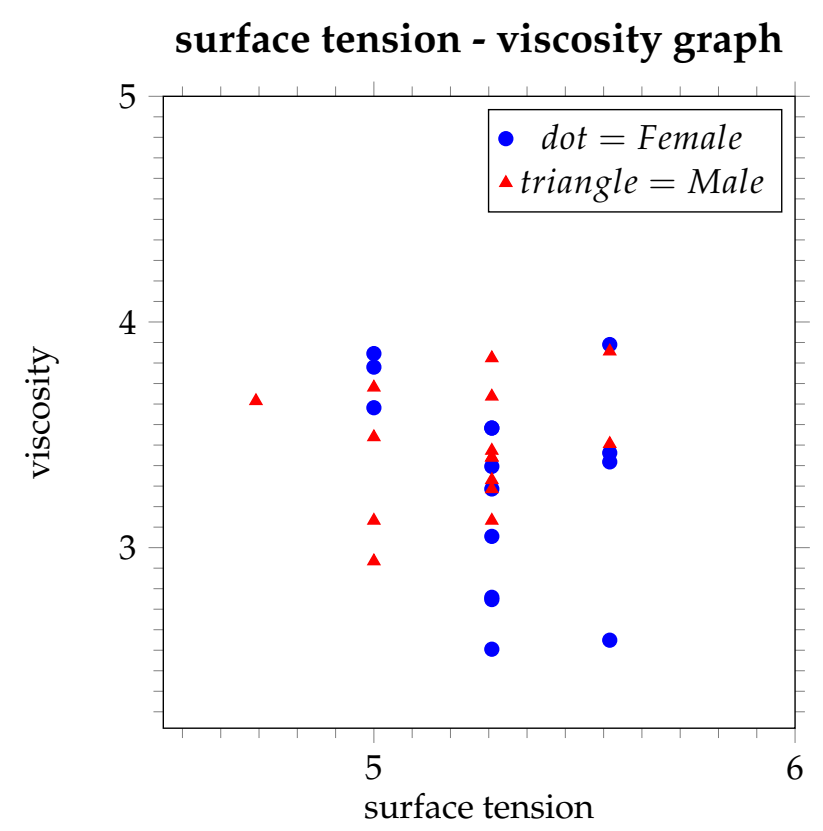

Figure 1: The above cross-plot depicts a relation between values of viscosity $\left(\cdot 10^{-3} \frac{\mathrm{P}}{\mathrm{m}}\right)$ and surface tension $\left(\cdot 10^{-2} \frac{\mathrm{N}}{\mathrm{m}}\right)$ 